\title{
REMARKS ON THE NONLINEAR STABILITY OF THE KURAMOTO MODEL WITH INERTIA
}

\author{
BY \\ YOUNG-PIL CHOI (Department of Mathematics, Imperial College London, London SW7 2AZ, \\ England), \\ SEUNG-YEAL HA (Department of Mathematical Sciences, Seoul National University, Seoul \\ 151-747, Republic of Korea), \\ AND \\ SE EUN NOH (Department of Mathematics, Myongji University, Yongin 449-728, Republic of \\ Korea)
}

Abstract. In this short note, we present an a priori nonlinear stability estimate for the Kuramoto model with finite inertia in $\ell^{\infty}$-norm under some a priori condition on the size of the phase diameter. As a direct corollary of our nonlinear stability estimate, we show that phase-locked states obtained in Choi, Ha, and Yun (2011) are orbital-stable in $\ell^{\infty}$-norm, which means that the perturbed phase-locked state approaches the phase-shift of the given phase-locked state. The phase-shift is explicitly determined by the averages of initial phase and frequency distribution and the strength of inertia $m$.

1. Introduction. The purpose of this paper is to present an elementary and simple proof for the nonlinear stability of the Kuramoto model with finite inertia. After $\mathrm{Ku}-$ ramoto introduced a simple mathematical model for the coupled limit cycles [8] from the two-dimensional complex Ginzburg-Landau equation, the Kuramoto model has become a prototype model for the collective synchronization phenomena ubiquitous in real systems ranging from physics to biology. One easy way to visualize the ensemble of an oscillator system with inertia is to view the ensemble as the set of point rotors moving on the circle $\mathbb{S}^{1} \subset \mathbb{C}^{1}$. Let $x_{i}=e^{\sqrt{-1} \theta_{i}}, \theta_{i} \in \mathbb{R}$, be the polar coordinate of the $i$-th rotator's position, and assume that all rotors are under the effect of inertia with strength $m$. In

Received September 13, 2013.

2000 Mathematics Subject Classification. Primary 92D25, 74A25, 76N10.

The first author was supported by the Basic Science Research Program through the NRF funded by the Ministry of Education, Science and Technology (2012R1A6A3A03039496).

The second author was supported by NRF grant 2011-0015388.

The third author was partially supported by the 2012 Research Fund of Myongji University.

E-mail address: young-pil.choi@imperial.ac.uk

E-mail address: syha@snu.ac.kr

WWW address: http://www.math.snu.ac.kr/ syha

E-mail address: senoh@mju.ac.kr 
this situation, the dynamics of the phase $\theta_{i}$ is governed by the system of the second-order ODEs in $\mathbb{R}^{N}$ :

$$
m \ddot{\theta}_{i}+\dot{\theta}_{i}=\Omega_{i}+\frac{K}{N} \sum_{j=1}^{N} \sin \left(\theta_{j}-\theta_{i}\right), \quad t>0, \quad i=1, \ldots, N
$$

Note that the R.H.S. of (1.1) is $2 \pi$-periodic; thus, the system (1.1) induces the dynamical system on $\mathbb{T}^{N}$. However, for our purpose, we treat (1.1) as a system on the Euclidean space $\mathbb{R}^{N}$ instead of $\mathbb{T}^{N}$. Without loss of generality, we assume that the natural frequencies $\Omega_{i}$ have a zero mean:

$$
\Omega_{c}:=\frac{1}{N} \sum_{i=1}^{N} \Omega_{i} \equiv 0
$$

The system (1.1) was first introduced by Ermentrout 7] as a phenomenological model for the slow synchronization phenomena of certain biological systems, e.g., fireflies of the Pteroptyx malaccae, and has been extensively studied in relation to the Josephson junction array for superconductors $[2,6,10,14,18$. For a detailed review of mathematical and physical results, we refer to [1,11-13. In Choi et al. 4], the authors studied the complete synchronization problem (see Definition 2.1) for (1.1), that is, to look for sufficient conditions leading to the complete synchronization in terms of initial configurations, inertia strength, coupling strength, etc. In fact, they analytically show that in the case of the large inertia $m K \gg 1$ some initial phase-frequency configurations have slower relaxation speed toward the phase-locked states than that of the Kuramoto model without inertia, which rigorously justifies Ermentrout's motivation for the model (1.1). The linear stabilities of the phase-locked state to (1.1) have been studied in [3, 9, 11, 12, in the absence of inertia. Thus a natural follow-up question that we may ask is whether the phase-locked states are nonlinearly stable, i.e., have robustness of phase-locked states. This question was addressed in [5] for the Kuramoto model without inertia, and the authors used the $\ell^{1}$-metric as a Lyapunov functional to establish the nonlinear stability of the phase-locked states without resorting to the linearization process.

The main result of this paper is the nonlinear stability estimate of the phase-locked states emerging from some class initial configurations whose existence has been guaranteed by the result of [4. For this, we use a phase-diameter which is equivalent to the $\ell^{\infty}$-norm as a Lyapunov functional and derive a second-order Gronwall's type inequality for the phase-diameter.

This paper after this Introduction consists of three sections. In Section 2, we briefly recall some basic definitions and mathematical structures of the system (1.1) and recall the existence of phase-locked states from initial phase-frequency configurations. In Section 3, we present the main result of this paper, the proof of a priori nonlinear stability of the system (1.1). As a direct corollary of our nonlinear stability result, we obtain the orbital stability of some class of phase-locked states. Finally, Section 4 is devoted to the summary and discussion of our main result. 
Notation. For $\theta=\left(\theta_{1}, \cdots, \theta_{N}\right), \Omega=\left(\Omega_{1}, \cdots, \Omega_{N}\right) \in \mathbb{R}^{N}$,

$$
\begin{gathered}
\|\theta\|_{\ell^{p}}:= \begin{cases}\left(\sum_{i=1}^{N}\left|\theta_{i}\right|^{p}\right)^{\frac{1}{p}}, & p \in[1, \infty), \\
\max _{1 \leq i \leq N}\left|\theta_{i}\right|, & p=\infty .\end{cases} \\
D(\theta):=\max _{1 \leq i, j \leq N}\left|\theta_{i}-\theta_{j}\right| \text { and } D(\Omega):=\max _{1 \leq i, j \leq N}\left|\Omega_{i}-\Omega_{j}\right| .
\end{gathered}
$$

Note that $D(\theta)$ and $D(\Omega)$ denote the diameters of the phase and frequency sets respectively.

2. Preliminaries. In this section, we briefly present basic definitions and recall the second-order Gronwall's inequality and existence of phase-locked states emerging from the limit of dynamical solutions to (1.1).

We rewrite the system (1.1) as a system of first-order ODEs for $\left(\theta_{i}, \omega_{i}:=\dot{\theta}_{i}\right)$ :

$$
\begin{aligned}
& \dot{\theta}_{i}=\omega_{i}, \quad t>0, \\
& \dot{\omega}_{i}=\frac{1}{m}\left(-\omega_{i}+\Omega_{i}+\frac{K}{N} \sum_{j=1}^{N} \sin \left(\theta_{j}-\theta_{i}\right)\right),
\end{aligned}
$$

and introduce macro-variables (center-of-mass frame) and micro-variables (fluctuations around macro-variables):

$$
\theta_{c}:=\frac{1}{N} \sum_{i=1}^{N} \theta_{i}, \quad \omega_{c}:=\frac{1}{N} \sum_{i=1}^{N} \omega_{i}, \quad \hat{\theta}_{i}:=\theta_{i}-\theta_{c}, \quad \hat{\omega}_{i}:=\omega_{i}-\omega_{c} .
$$

Then, macro-variables and micro-variables satisfy

$$
\dot{\theta}_{c}=\omega_{c}, \quad \dot{\omega}_{c}=-\frac{\omega_{c}}{m},
$$

and

$$
\dot{\hat{\theta}}_{i}=\hat{\omega}_{i}, \quad m \dot{\hat{\omega}}_{i}=-\hat{\omega}_{i}+\hat{\Omega}_{i}+\frac{K}{N} \sum_{j=1}^{N} \sin \left(\hat{\theta}_{j}-\hat{\theta}_{i}\right) .
$$

Note that the macroscopic variables $\left(\theta_{c}, \omega_{c}\right)$ are given by the following formula:

$$
\theta_{c}(t)=\theta_{c}(0)+m \omega_{c}(0)\left(1-e^{-\frac{t}{m}}\right), \quad \omega_{c}(t)=e^{-\frac{t}{m}} \omega_{c}(0) .
$$

Thus, macro-variables converge toward some constant states that are determined by $m$ and initial configurations:

$$
\lim _{t \rightarrow \infty}\left(\theta_{c}(t), \omega_{c}(t)\right)=\left(\theta_{c}(0)+m \omega_{c}(0), 0\right) .
$$

We next recall several definitions for the phase-locked state and its orbital stability as follows. 
Definition 2.1. (1) Let $\theta(t)=\left(\theta_{1}(t), \cdots, \theta_{N}(t)\right)$ be the dynamical solution of the system (1.1). Then the dynamical solution asymptotically approaches the completely synchronized states if and only if the transversal frequency differences $\dot{\theta}_{i}-\dot{\theta}_{j}$ go to zero as $t \rightarrow \infty$ :

$$
\lim _{t \rightarrow \infty}\left|\dot{\theta}_{i}(t)-\dot{\theta}_{j}(t)\right|=0, \quad 1 \leq i, j \leq N .
$$

(2) A phase locked solution $\theta_{e}$ to the system (1.1) - (1.2) is an equilibrium solution to the system of ODEs, (1.1) - (1.2):

$$
\Omega_{i}+\frac{K}{N} \sum_{i=1}^{N} \sin \left(\theta_{e j}-\theta_{e i}\right)=0, \quad \dot{\theta}_{e i}=0, \quad i=1, \cdots, N .
$$

(3) The phase locked state $\theta_{e}$ for (1.1) - (1.2) is orbital-stable in norm $\|\cdot\|$ if and only if for any small perturbation $\theta_{0}$ of $\theta_{e}$, the dynamical solution $\theta(t)$ with initial data $\theta_{0}$ asymptotically converges to the phase-shift of $\theta_{e}$ in the norm $\|\cdot\|$ :

$$
\exists \beta \in \mathbb{R}^{N} \quad \text { such that } \quad \lim _{t \rightarrow \infty}\left\|\theta(t)-\left(\theta_{e}+\beta \mathbb{I}_{N}\right)\right\|=0, \quad \mathbb{I}_{N}:=(1, \cdots, 1) .
$$

\section{REMARK 2.2.}

(1) When the natural frequency does not have mean zero, the phase-locked state is a traveling wave with a constant phase velocity $\Omega_{c}$.

(2) The phase-locked state employed in Definition 2.1 is taken in a strong sense. We may relax the definition of (weak) phase-locked states as follows. The dynamical solution $\theta=\theta(t)$ is (weakly) phase-locked if and only if there exist positive constants $C_{*}, C^{*} \geq 0$ independent of $t$ satisfying

$$
C_{*} \leq\left|\theta_{i}(t)-\theta_{j}(t)\right| \leq C^{*}, \quad t \geq 0 .
$$

Below, we review the second-order Gronwall's inequality and existence of phase-locked states. Consider the second-order Gronwall's inequality:

$$
\begin{aligned}
& a \ddot{y}+b \dot{y}+c y \leq 0, \quad t>0, \\
& y(0)=y_{0}, \quad \dot{y}(0)=y_{1},
\end{aligned}
$$

where $a>0, b$ and $c$ are constants.

Lemma 2.3. 4] Let $y=y(t)$ be a nonnegative $C^{2}$-function satisfying the differential inequality (2.6). Then we have

$$
y(t) \leq \begin{cases}y_{0} e^{-\nu_{1} t}+a \frac{e^{-\nu_{2} t}-e^{-\nu_{1} t}}{\sqrt{b^{2}-4 a c}}\left(y_{1}+\nu_{1} y_{0}\right), & b^{2}-4 a c>0, \\ e^{-\frac{b}{2 a} t}\left[y_{0}+\left(\frac{b}{2 a} y_{0}+y_{1}\right) t\right], & b^{2}-4 a c \leq 0,\end{cases}
$$

where decay exponents $\nu_{1}$ and $\nu_{2}$ are given as follows:

$$
\nu_{1}:=\frac{b+\sqrt{b^{2}-4 a c}}{2 a}, \quad \nu_{2}:=\frac{b-\sqrt{b^{2}-4 a c}}{2 a} .
$$

This lemma will be used crucially in the next section. Finally, we discuss two frameworks which guarantee the existence of phase-locked states.

- Framework A: (Small inertia regime) 
(1) The strength of coupling $K$ and the magnitude of inertia $m$ satisfy

$$
0<D(\Omega)<K, \quad m K<\frac{D^{\infty}}{4 \sin D^{\infty}},
$$

where $D^{\infty} \in\left(0, \frac{\pi}{2}\right)$ is the root of the following trigonometric equation:

$$
\sin x=\frac{D(\Omega)}{K} .
$$

(2) An initial configuration of $\left(\theta_{0}, \omega_{0}\right)$ satisfies

$$
0<\max \left\{D\left(\theta_{0}\right), D\left(\theta_{0}\right)+\left.2 m \dot{D}(\theta(t))\right|_{t=0}\right\}<D^{\infty} .
$$

- Framework B: (Large inertia regime)

(1) The strength of coupling $K$ and the magnitude of inertia $m$ satisfy

$$
0<D(\Omega)<\frac{\pi}{8 m}, \quad m K>\frac{\pi}{8} .
$$

(2) An initial configuration of $\left(\theta_{0}, \omega_{0}\right)$ satisfies

$$
0<\max \left\{D\left(\theta_{0}\right), D\left(\theta_{0}\right)+\left.2 m \dot{D}(\theta(t))\right|_{t=0}\right\}<4 m D(\Omega) .
$$

We also note that for $D^{\infty} \in\left(0, \frac{\pi}{2}\right)$,

$$
\frac{D^{\infty}}{4 \sin D^{\infty}}<\frac{\pi}{8}
$$

Theorem 2.4 (4]). Under either Framework A or Framework B, there exists a phaselocked state $\theta_{e}$ to the system (1.1) - (1.2), and its phase-diameter is smaller than $\frac{\pi}{2}$.

Remark 2.5. 1) The conditions of Framework A and Framework B are independent of the system size $N$. Hence, our result can be lifted to the kinetic regime via the thermodynamic limit.

2) Under both Framework A and Framework B, the initial phase-frequency configuration $\left(\theta_{0}, \omega_{0}\right)$ evolves toward the asymptotic phase-locked state $\left(\theta_{e}, 0\right)$ :

$$
\Omega_{i}+\frac{K}{N} \sum_{i=1}^{N} \sin \left(\theta_{e j}-\theta_{e i}\right)=0, \quad \sup _{t \geq 0} D(\theta(t))<\frac{\pi}{2} .
$$

3. A priori nonlinear stability. In this section, we present a nonlinear stability of the Kuramoto model with inertia in the $\ell^{\infty}$-norm.

Theorem 3.1. Let $\theta$ and $\tilde{\theta}$ be two solutions to the system (1.1) - (1.2) satisfying the a priori conditions:

$$
\begin{aligned}
& \text { (i) } D_{\infty}^{a v}:=\sup _{t \geq 0}\left(\frac{D(\theta(t))+D(\tilde{\theta}(t))}{2}\right)<\frac{\pi}{2} . \\
& \text { (ii) } \sum_{i=1}^{N} \theta_{i}(t)=\sum_{i=1}^{N} \tilde{\theta}_{i}(t), \quad t \geq 0 .
\end{aligned}
$$


Then for any $\varepsilon \in\left(0, \frac{1}{2 m}\right)$, there exists a constant $\Lambda=\Lambda(\varepsilon)>0$ dependent only on $\varepsilon$ such that

$$
\|\theta(t)-\tilde{\theta}(t)\|_{\ell^{\infty}} \leq C\left(\theta_{0}, \tilde{\theta}_{0}\right) e^{-\Lambda(\varepsilon) t}, \quad t \geq 0,
$$

where $C\left(\theta_{0}, \tilde{\theta}_{0}\right)$ is a positive constant depending only on $\theta_{0}$ and $\tilde{\theta}_{0}$.

Proof. Let $\theta$ and $\bar{\theta}$ be two solutions satisfying the a priori conditions (3.1) - (3.2), and set

$$
\alpha_{i}:=\theta_{i}-\tilde{\theta}_{i}, \quad \alpha_{M}:=\max _{1 \leq i \leq N} \alpha_{i}, \quad \alpha_{m}:=\min _{1 \leq i \leq N} \alpha_{i}, \quad D(\alpha):=\alpha_{M}-\alpha_{m}
$$

Then it follows from (3.1) and (3.2) that $\alpha=\left(\alpha_{1}, \cdots, \alpha_{N}\right)$ satisfies

$$
\sum_{i=1}^{N} \alpha_{i}(t)=0, \quad D(\alpha(t))<\pi, \quad t \geq 0 .
$$

Since $\|\alpha\|_{\ell_{\infty}} \leq D(\alpha)$, it suffice to show that $D(\alpha)$ decays exponentially fast to get the desired estimate. Note that $\alpha_{i}$ satisfies

$$
m \ddot{\alpha}_{i}+\dot{\alpha}_{i}=\frac{2 K}{N} \sum_{j=1}^{N} \cos \left(\frac{\theta_{j}-\theta_{i}}{2}+\frac{\tilde{\theta}_{j}-\tilde{\theta}_{i}}{2}\right) \sin \left(\frac{\alpha_{j}-\alpha_{i}}{2}\right) .
$$

- Step A (Derivation of Gronwall's inequality for $D(\alpha)$ ): It follows from (3.4) that

$$
\begin{aligned}
m \ddot{\alpha}_{M}+\dot{\alpha}_{M} & =\frac{2 K}{N} \sum_{j=1}^{N} \cos \left(\frac{\theta_{j}-\theta_{i}}{2}+\frac{\tilde{\theta}_{j}-\tilde{\theta}_{i}}{2}\right) \sin \left(\frac{\alpha_{j}-\alpha_{M}}{2}\right) \\
& \leq\left(\frac{K \sin 2 D_{\infty}^{a v}}{D_{\infty}^{a v} N}\right) \sum_{j=1}^{N}\left(\frac{\alpha_{j}-\alpha_{M}}{2}\right) \\
& =-\frac{K \sin 2 D_{\infty}^{a v}}{2 D_{\infty}^{a v}} \alpha_{M},
\end{aligned}
$$

where we used the fact that $-\pi<\alpha_{j}-\alpha_{M} \leq 0$ to find

$$
\sum_{i=1}^{N} \alpha_{i}=0, \quad \sin \left(\frac{\alpha_{j}-\alpha_{M}}{2}\right) \leq\left(\frac{\sin D_{\infty}^{a v}}{D_{\infty}^{a v}}\right)\left(\frac{\alpha_{j}-\alpha_{M}}{2}\right) .
$$

Similarly, we find

$$
m \ddot{\alpha}_{m}+\dot{\alpha}_{m} \geq-\frac{K \sin 2 D_{\infty}^{a v}}{2 D_{\infty}^{a v}} \alpha_{m} .
$$

We combine the estimates (3.5) and (3.6) to find

$$
m \ddot{D}(\alpha)+\dot{D}(\alpha)+\bar{K} D(\alpha) \leq 0, \quad \bar{K}:=\frac{K \sin 2 D_{\infty}^{a v}}{2 D_{\infty}^{a v}} .
$$

- Step B (Applying Lemma 2.3): We apply Lemma 2.3 for (3.7) to obtain

$$
D(\alpha(t)) \leq \begin{cases}D\left(\alpha_{0}\right) e^{-\mu_{1} t}+m \frac{e^{-\mu_{2} t}-e^{-\mu_{1} t}}{\sqrt{1-4 m \bar{K}}}\left(\dot{D}\left(\alpha_{0}\right)+\mu_{1} D\left(\alpha_{0}\right)\right), & 1-4 m \bar{K}>0, \\ e^{-\frac{t}{2 m}}\left[D\left(\alpha_{0}\right)+\left(\frac{1}{2 m} D\left(\alpha_{0}\right)+\dot{D}\left(\alpha_{0}\right)\right) t\right], & 1-4 m \bar{K} \leq 0,\end{cases}
$$

where

$$
\mu_{1}=\frac{1+\sqrt{1-4 m \bar{K}}}{2 m}, \quad \mu_{2}=\frac{1-\sqrt{1-4 m \bar{K}}}{2 m} .
$$


This yields the desired result.

REMARK 3.2. 1) We briefly discuss the a priori conditions (3.1) - (3.2). In Frameworks $\mathrm{A}$ and $\mathrm{B}$, the phase-diameter of dynamical solutions is always less than $\frac{\pi}{2}$. Thus, the dynamical solutions in [4] satisfy (3.1). As long as

$$
\theta_{c}(0)=\tilde{\theta}_{c}(0), \quad \omega_{c}(0)=\tilde{\omega}_{c}(0)
$$

the condition (3.2) is satisfied.

2) For the Kuramoto model without inertia $(m=0)$, the nonlinear stability estimate in the $\ell^{1}$-norm has been established in $[\underline{5}$, and it is also shown that the decay rate in the large time regime is bounded not only above but also below.

3) As a direct application of Theorem 3.1, we will see that all phase-locked states emerging from initial configurations in Frameworks A and B are unique up to phaseshift.

Consider the collection $\mathcal{P}$ of all phase-locked states emerging from dynamical solutions as an asymptotic limit, whose existence is guaranteed by Theorem 2.4. Of course, the set $\mathcal{P}$ is the proper subset of all possible phase-locked states for the system (1.1) - (1.2), and it follows that phase-locked states in $\mathcal{P}$ have a diameter strictly less than $\frac{\pi}{2}$. As a direct corollary of Theorem 3.1, we see that the phase-locked states $\theta_{e} \in \mathcal{P}$ are orbitally stable.

Corollary 1. Let $\theta_{e}$ and $\left(\theta_{0}, \omega_{0}\right)$ be a phase-locked state in $\mathcal{P}$ and the perturbation of $\left(\theta_{e}, 0\right)$ respectively in phase-frequency space $\mathbb{R}^{N} \times \mathbb{R}^{N}$ satisfying either Framework A or Framework B. Then the dynamical solution $\theta=\theta(t)$ asymptotically approaches the phase-shift of $\theta_{e}$; i.e., $\theta_{e}$ is orbital-stable:

$$
\lim _{t \rightarrow \infty}\left\|\theta(t)-\left(\theta_{e}+\gamma \mathbb{I}_{N}\right)\right\|_{\ell^{\infty}}=0,
$$

where the constant phase-shift $\gamma$ is explicitly given by

$$
\gamma:=\theta_{c}(0)+m \omega_{c}(0)-\theta_{e c} .
$$

Proof. We set

$$
\alpha(t):=\theta(t)-\theta_{e} .
$$

Then its macro-variable $\alpha_{c}(t)$ converges to the constant state:

$$
\lim _{t \rightarrow \infty} \alpha_{c}(t)=\theta_{c}(0)+m \omega_{c}(0)-\theta_{e c}=: \gamma,
$$

and the diameter of $\alpha(t)$ shrinks exponentially fast as we saw in the proof of Theorem 3.1. We now combine these two facts through the triangle inequality to obtain

$$
\begin{aligned}
\left\|\theta(t)-\left(\theta_{e}+\gamma \mathbb{I}_{N}\right)\right\|_{\ell^{\infty}} & =\left\|\alpha(t)-\gamma \mathbb{I}_{N}\right\|_{\ell^{\infty}} \\
& \leq\left\|\alpha(t)-\alpha_{c}(t) \mathbb{I}_{N}\right\|_{\ell^{\infty}}+\left\|\left(\alpha_{c}(t)-\gamma\right) \mathbb{I}_{N}\right\|_{\ell^{\infty}} \\
& \leq 2 D(\alpha(t))+\left\|\left(\alpha_{c}(t)-\gamma\right) \mathbb{I}_{N}\right\|_{\ell^{\infty}},
\end{aligned}
$$

where we used (3.8). This completes the proof. 
REMARK 3.3. Corollary 1 implies that a phase-locked state with the phase-diameter $D(\theta)$ strictly less than $\frac{\pi}{2}$ is unique up to the phase-shift. Let $\theta^{\infty}$ and $\tilde{\theta}^{\infty}$ be the two phase-locked states emerging from initial data $\left(\theta_{0}, \omega_{0}\right)$ and $\left(\tilde{\theta}_{0}, \tilde{\omega}_{0}\right)$ such that

$$
D\left(\theta^{\infty}\right), D\left(\tilde{\theta}^{\infty}\right)<\frac{\pi}{2} .
$$

Then, by the same argument as in Theorem $3.1, \theta^{\infty}$ and $\tilde{\theta}^{\infty}$ are congruent in the sense that

$$
\theta^{\infty}-\tilde{\theta}^{\infty}=\left(\theta_{c}(0)-\tilde{\theta}_{c}(0)+m\left(\omega_{c}(0)-\tilde{\omega}_{c}(0)\right)\right) \mathbb{I}_{N}
$$

4. Conclusion. We presented a simple proof for the nonlinear stability of some class of phase-locked states to the Kuramoto model with finite inertia in the $\ell^{\infty}$-norm. In the earlier work [4, the existence of phase-locked states is established via the timeasymptotic approach; i.e., instead of solving the above nonlinear system (1.1) directly, the phase-locked states are obtained as an asymptotic limit of time-dependent solutions to the system (1.1). This time-asymptotic approach can reveal the fine structure of the phase-locked states (see [5] for the original Kuramoto model). In this paper, we have adopted the same framework ideas employed in the aforementioned work, where the existence of phase-locked states was only investigated, and we showed that the phaselocked states whose existence is guaranteed by [4] are orbitally $\ell^{\infty}$-stable in the sense that their small perturbations lead to the relative phase-shift from the original phaselocked state. Based on this fact, we claim that the phase-locked state has in some sense a robust structure. At present, we cannot provide a complete classification for the stability of phase-locked states to the system (1.1), such as clear answers to the following questions: For a given $m, K$ and $\left\{\Omega_{i}\right\}$, how many phase-locked states exist up to phase-shift? Are all phase-locked states orbitally stable? We leave these intriguing issues for future works to investigate, as well as the nature of phase transitions in the Kuramoto oscillators with finite inertia.

\section{REFERENCES}

[1] J. A. Acebrón, L .L. Bonilla, C. J. P. Pérez Vicente, F. Ritort, R. Spigler, The Kuramoto model: A simple paradigm for synchronization phenomena, Rev. Mod. Phys. 77 (2005), 137-185.

[2] J. A. Acebrón, R. Spigler, Adaptive frequency model for phase-frequency synchronization in large populations of globally coupled nonlinear oscillators, Phys. Rev. Lett. 81 (1998), 2229-2332.

[3] D. Aeyels, J. Rogge, Existence of partial entrainment and stability of phase locking behavior of coupled oscillator, Prog. Theor. Phys. 112 (2004), 921-942.

[4] Young-Pil Choi, Seung-Yeal Ha, and Seok-Bae Yun, Complete synchronization of Kuramoto oscillators with finite inertia, Phys. D 240 (2011), no. 1, 32-44, DOI 10.1016/j.physd.2010.08.004. MR2740100 (2012b:34093)

[5] Young-Pil Choi, Seung-Yeal Ha, Sungeun Jung, and Yongduck Kim, Asymptotic formation and orbital stability of phase-locked states for the Kuramoto model, Phys. D 241 (2012), no. 7, 735-754, DOI 10.1016/j.physd.2011.11.011. MR2897541

[6] B. C. Daniels, S. T. Dissanayake, B. R. Trees, Synchronization of coupled rotators: Josephson junction ladders and the locally coupled Kuramoto model, Phys. Rev. E. 67 (2003), 026216.

[7] B. Ermentrout, An adaptive model for synchrony in the firefly Pteroptyx malaccae, J. Math. Biol. 29 (1991), no. 6, 571-585, DOI 10.1007/BF00164052. MR.1118757

[8] Yoshiki Kuramoto, Self-entrainment of a population of coupled non-linear oscillators (Kyoto Univ., Kyoto, 1975), Lecture Notes in Phys., 39, Springer, Berlin, 1975, pp. 420-422. MR0676492 $(58$ \#32705) 
[9] Renato E. Mirollo and Steven H. Strogatz, The spectrum of the locked state for the Kuramoto model of coupled oscillators, Phys. D 205 (2005), no. 1-4, 249-266, DOI 10.1016/j.physd.2005.01.017. MR2167156 (2006d:34080)

[10] K. Park, M. Y. Choi, Synchronization in networks of superconducting wires, Phys. Rev. B 56 (1997), 387-394.

[11] Steven H. Strogatz and Renato E. Mirollo, Stability of incoherence in a population of coupled oscillators, J. Statist. Phys. 63 (1991), no. 3-4, 613-635, DOI 10.1007/BF01029202. MR1115806 (92h:34077)

[12] S. H. Strogatz, R. E. Mirollo, Splay states in globally coupled Josephson arrays: Analytical prediction of Floquet multipliers, Phys. Rev. E 47 (1993), 220-227.

[13] James W. Swift, Steven H. Strogatz, and Kurt Wiesenfeld, Averaging of globally coupled oscillators, Phys. D 55 (1992), no. 3-4, 239-250, DOI 10.1016/0167-2789(92)90057-T. MR.1156731(92m:34085)

[14] K. Wiesenfeld, R. Colet, S. H. Strogatz, Frequency locking in Josephson arrays: Connection with the Kuramoto model, Phys. Rev. E 57 (1988), 1563-1569.

[15] K. Wiesenfeld, R. Colet, S. H. Strogatz, Synchronization transitions in a disordered Josephson series arrays, Phys. Rev. Lett. 76 (1996), 404-407.

[16] S. Watanabe and J. W. Swift, Stability of periodic solutions in series arrays of Josephson junctions with internal capacitance, J. Nonlinear Sci. 7 (1997), no. 6, 503-536, DOI 10.1007/s003329900038. MR.1474640 (98f:34051)

[17] S. Watanabe, S. H. Strogatz, Constants of motion for superconducting Josephson arrays, Physica D 74 (1994), 197-253.

[18] K. Wiesenfeld, J. W. Swift, Averaged equations for Josephson junction series arrays, Phys. Rev. E 51 (1995), 1020-1025. 